\title{
Serum miR-375 Levels Are Closely Related to Disease Progression from HBV Infection to HBV-Related Hepatocellular Carcinoma
}

\author{
Weilu Zhang, ${ }^{1}$ Ting Fu, ${ }^{1}$ Zhenjun Guo, ${ }^{2}$ Ye Zhang, ${ }^{3}$ Lei Zhang, ${ }^{1}$ Haixia Su, ${ }^{1}$ Yong Long, \\ Zhaohua Ji, ${ }^{1}$ Yongping Yan ${ }^{1},{ }^{1}$ and Zhongjun Shao $\mathbb{1 D}^{1}$ \\ ${ }^{1}$ Department of Epidemiology, Ministry of Education Key Lab of Hazard Assessment and Control in Special \\ Operational Environment, School of Public Health, The Air Force Medical University, No. 17, Changle West RD, \\ Xi'an 710032, China \\ ${ }^{2}$ Shaanxi Provincial People's Hospital, No. 256, Youyi West RD, Xi'an 710068, China \\ ${ }^{3}$ Center for Infectious Diseases, Tangdu Hospital, Air Force Medical University, 569 Xinsi Rd, Xi'an 710032, China
}

Correspondence should be addressed to Yongping Yan; 315987098@qq.com and Zhongjun Shao; zhongjunshao@126.com

Received 3 January 2020; Revised 10 March 2020; Accepted 16 March 2020; Published 21 April 2020

Academic Editor: Xiaofeng Fan

Copyright (C) 2020 Weilu Zhang et al. This is an open access article distributed under the Creative Commons Attribution License, which permits unrestricted use, distribution, and reproduction in any medium, provided the original work is properly cited.

Background. There is an urgent need to identify ideal serological biomarkers that not only are closely related to disease progression from hepatitis B virus (HBV) infection to hepatocellular carcinoma (HCC) but also have high specificity and sensitivity. We conducted this study to analyze whether miR-375 has a potential value in the early prediction of the progression from HBVrelated hepatitis or cirrhosis to HCC. Methods. A total of 177 participants were enrolled. Receiver operating characteristic (ROC) curve was used to evaluate the predictive capability of selected miR-375 for HBV-HCC. We upregulated the miR-375 expression in HepG2, HepG2.2.15, and HepAD38 cells to determine its effect on cellular proliferation and migration, in vitro using Cell Counting Kit-8 (CCK-8) assays. Results. Serum miR-375 levels decreased in order from healthy controls to chronic hepatitis $\mathrm{B}(\mathrm{CHB})$ without cirrhosis, followed by cirrhosis, and finally, HBV-HCC patients. miR-375 levels were significantly lower in HBeAg-positive and HBV DNA-positive patients than negative $(P<0.05)$ and significantly lower in patients with elevated alpha-fetoprotein (AFP) and carcinoembryonic antigen (CEA) than normal levels $(P<0.05)$. miR-375 might be a biomarker for HBV-HCC, with a high area under the curve (AUC) of 0.838 (95\% confidence interval (CI) 0.780 to 0.897 ; sensitivity: $73.9 \%$; specificity: $93.0 \%)$. The AUC (0.768 vs. 0.584$)$ and sensitivity (93.8\% vs. $75.0 \%)$ for miR-375 were higher than those for AFP. The overexpression of miR-375 noticeably inhibited proliferation and migration in HepG2, HepG2.2.15, and HepAD38, especially in HepG2.2.15 and HepAD38, which are stably infected with HBV. Conclusions. Serum miR-375 levels are closely related to disease progression from HBV-related hepatitis or cirrhosis to HCC.

\section{Background}

It is estimated that almost $240,000,000$ people are chronically infected with hepatitis B virus (HBV). Annually, HBV infection accounts for approximately 1 million deaths [1]. Hepatocellular carcinoma (HCC) is the major form of primary liver tumor, representing the third leading cause of cancerrelated death worldwide. Nearly half of HCC cases and deaths are estimated to occur in China due to the high prevalence of HBV infection. The cancer statistics in China show that the mortality due to $\mathrm{HCC}$ is $42.21 / 100,000$ [2]. Patients with HCC have a poor prognosis, with a 5-year survival of approximately $5 \%$ because of diagnosis at late stages and limited treatment options [3]. Therefore, it is important to predict the progression from HBV-related hepatitis or cirrhosis to HCC.

Recently, many biomarkers have been widely used for HCC diagnosis, such as alpha-fetoprotein (AFP) [4], suppressor with morphogenetic effect on genitalia-1 (SMG-1) $[5,6]$, Golgi membrane protein 1 (GOLM1) [7], BarH-like 
Homeobox 2 (Barx2) [8], des- $\gamma$-carboxy prothrombin (DCP) [9], glypican-3 [10], cytokeratin-19 [11], Golgi protein-73 [12], osteopontin [13], miR-122, and PIVKA-II [14]. Nevertheless, most of these proteins are usually detected after HCC occurrence and lack sensitivity and specificity [15]. Moreover, these markers are not specific for HBV-HCC. Hence, it is still difficult to predict disease progression from healthy liver, chronic hepatitis $\mathrm{B}$ infection $(\mathrm{CHB})$ without cirrhosis, and $\mathrm{CHB}$ with cirrhosis to HBV-HCC. Therefore, there is an urgent need to identify ideal serological biomarkers that not only are closely related to disease progression from $\mathrm{HBV}$ infection to HCC but also have high specificity and sensitivity.

MicroRNAs (miRNAs) are known to play fundamental roles in the regulation of many oncogenes or tumor suppressors and are considered potential diagnostic biomarkers for cancer detection $[16,17]$. miR-375 is encoded by the chromosomal region $2 \mathrm{q} 35$ in humans. Some studies have reported that miR-375 is downregulated including HCC [18-21]. Research has shown that miR-375 could be used as a serological marker for HCC [22]. When employed as biomarkers, miR-25, miR-375, and let-7f could separate HCC patients from controls. The area under the receiver operating characteristic (ROC) curve (AUC) for miR-375 for HCC prediction was 0.96 (specificity: 96\%; sensitivity: 100\%) [23]. Mechanistic studies have shown that miR-375 inhibited proliferation, migration, and invasion in Hep3B and Huh7 cells [24]. However, there are very few studies on the relationship between miR-375 and HBV-HCC in patients or in HBVinfected cell lines. Moreover, there is a lack of epidemiological studies on the association of serum miR-375 expression with disease progression from healthy liver, $\mathrm{CHB}$ without cirrhosis, and cirrhosis to HBV-HCC.

In this study, we determined serum miR-375 levels in healthy controls and in patients with $\mathrm{CHB}$ without cirrhosis, cirrhosis, or HBV-HCC and analyzed the correlation between miR-375 and disease progression, HBV-related serological markers, and clinical features. Then, we upregulated miR-375 expression in HepG2, HepG2.2.15, and HepAD38 cells to determine its effect on cellular proliferation and migration in vitro. Additionally, we evaluated the diagnostic value of serum miR-375 as an ideal serological biomarker for the prediction of HBV-related hepatitis or cirrhosis to HCC.

\section{Materials and Methods}

2.1. Subjects. From August 2015 to August 2017, healthy individuals and patients with $\mathrm{HBV}$-related diseases who were older than 18 years were recruited from the Department of Hepatobiliary Surgery, Xijing Hospital, and the Center of Infectious Diseases, Tangdu Hospital, Xi'an City, Shaanxi Province, China. All patients were positive for HBsAg, and none of the patients had any other type of liver disease, such as chronic hepatitis $\mathrm{C}$ infection, alcoholic liver disease, autoimmune liver disease, or metabolic liver disease. The diagnosis of HCC and cirrhosis was histopathologically confirmed. $\mathrm{CHB}$ in the liver was caused by persistent infection with $\mathrm{HBV}$. The diagnostic criteria included the following four points: (1) $\mathrm{HBsAg}^{+}>6$ months, (2) serum $\mathrm{HBV}$ DNA > 20,000 IU/mL ( $10^{5}$ copies/mL), (3) persistent or intermittent elevation in alanine transaminase (ALT)/aspartate aminotransferase (AST) levels, and (4) liver biopsy showing chronic hepatitis with moderate or severe necroinflammation. All the healthy controls were from the medical examination center, and they were not found to have any disease after physical examination.

A standard questionnaire was used by trained investigators to collect information from face-to-face interviews, pathology reports, and medical records. The questionnaire recorded the following information: basic demographic characteristics of the subjects, family history of HBV infection, cause of disease, tumor pathology, and clinical indicators of cancer. Blood samples for miRNA detection were collected in tubes and processed within $1 \mathrm{~h}$ of collection. The blood samples were centrifuged at $12,000 \mathrm{~g}$ for $10 \mathrm{~min}$ at $4^{\circ} \mathrm{C}$ to spin down blood cells, and the supernatants were transferred into microcentrifuge tubes, followed by a second centrifugation at $12,000 \mathrm{~g}$ for $10 \mathrm{~min}$ at $4^{\circ} \mathrm{C}$. The supernatants were transferred to RNase-free tubes and stored at $-80^{\circ} \mathrm{C}$.

2.2. Ethics, Consent, and Permissions. The Ethics Committee of the Air Force Medical University approved the study protocol. All participants were fully informed of the details of research study, and the participants or legal guardians of the participants signed written informed consent before inclusion in the study.

2.3. Serological Examination of $H B V$. Blood samples of participants were obtained by forearm venipuncture. The serum samples were laboratory tested after collection. The same lot of enzyme-linked immunosorbent assay (ELISA) reagents from Wantai Production Company (Beijing, China) was used for the initial testing of $\mathrm{HBsAg}, \mathrm{HBeAg}$, anti-HBs, anti-HBe, and anti-HBc. All specimens were evaluated for the presence of HBV DNA by using a diagnostic kit (for PCR-based fluorescence probing) according to the instructions of the manufacturer (Roche Molecular Systems). Viral loads higher than a linear range were determined by dilution as recommended by the manufacturer.

\subsection{Detection of miR-375 via Real-Time Quantitative RT- PCR}

2.4.1. RNA Isolation. Total RNA was isolated from serum samples using TRIzol reagent (Invitrogen, USA). Briefly, $250 \mu \mathrm{L}$ serum samples and all cell lines were used to extract total RNA. Each sample was eluted in $50 \mu \mathrm{L}$ of RNase-free water. The concentration and purification of RNA were spectrophotometrically determined by measuring its optical density (OD, A260/280>2.0 and A260/230>1.8) using a NanoDrop ND-2000 Spectrophotometer (Thermo Scientific Wilmington, DE, USA).

2.4.2. RNA Reverse Transcription and $q R T-P C R$. The primers used for Polymerase Chain Reaction (PCR) were as follows: miR-375 forward primer ( $5^{\prime}$-TTTGTTCGTTCGGCTCGC$\left.3^{\prime}\right)$ and reverse primer and U6 ( $5^{\prime}$-CGCTTCGGCAGCAC ATATAC- $3^{\prime}$ ) purchased from TaKaRa (Dalian, China). We 
used U6 as an endogenous control for both serum and cells. Reverse transcription of miRNAs was performed with the SYBR PrimeScript miRNA RT-PCR Kit (TaKaRa, Dalian, China). The expression of mature miRNAs was determined using miRNA-specific qRT-PCR (TaKaRa, Dalian, China). Fluorescence was automatically detected during amplification, and the melting curve for the product was obtained. The comparative cycle threshold $(\mathrm{Ct})$ method was used for calculation. And the $2^{-\Delta \mathrm{Ct}}$ method was used to calculate the relative levels of specific molecules [25].

2.5. Cell Culture. The following HCC cell lines were used in this study: HepG2, HepG2.2.15, and HepAD38. These cell lines were cultured in Dulbecco's modified Eagle's medium (DMEM, HyClone, USA) supplemented with $10 \%$ fetal bovine serum (FBS, Gibco, USA) and $380 \mathrm{mg} / \mathrm{L}$ G418 sulfate (Promega, USA) at $37^{\circ} \mathrm{C}$ in an atmosphere with $5 \% \mathrm{CO}_{2}$. HepAD38 cells, which are a variant of HepG2 cells, express the HBV genome under the control of a tetracycline- (Tet-) off promoter.

2.6. Cell Transfection. miR-375 mimics and negative control (NC) oligonucleotides were designed and synthesized by GenePharma (Shanghai, China). The sequences were as follows: miR-375 mimic, $5^{\prime}$-UUUGUUCGUUCGGCUCGCG UGA- ${ }^{\prime}$ and random miRNA mimics (NC), $5^{\prime}$-UUCUCC GAACGUGUCACGUTT-3' ${ }^{\prime}$ miR-375 mimics were transfected into cells at a final oligonucleotide concentration of $20 \mathrm{nmol} / \mathrm{L}$. Transfection was performed with Lipofectamine 2000 reagent (Invitrogen, CA, USA) following the manufacturer's protocol. Briefly, cells were trypsinized, counted, and seeded in plates on the day before transfection to ensure suitable cell confluency on the day of transfection.

2.7. Proliferation Assay. The Cell Counting Kit-8 (CCK-8) assay was carried out to assess cell viability. HepG2 cells, HepG2.2.15 cells, and HepAD38 were seeded in 96-well culture plates at $2 \times 10^{5}$ cells per well. The next day, the cells were transfected with miR-375 mimics or NC as above. Then, $10 \mathrm{~mL}$ of CCK- 8 reagent was added to each well before transfection and at $24 \mathrm{~h}, 48 \mathrm{~h}$, or $72 \mathrm{~h}$ after transfection. Cell proliferation rates were determined by the measurement of optical density (OD) at $490 \mathrm{~nm}$ via a microplate reader (Bio-Rad).

2.8. Statistical Analysis. RT-PCR was performed in triplicate, and average $\mathrm{Ct}$ values were calculated. The average expression level of miR-375 in all samples was normalized using U6 as the reference, and the $2^{-\Delta \mathrm{Ct}}$ method $(\Delta \mathrm{Ct}=\mathrm{miR}-375$ Ct-U6 Ct) was used to express the level of miR-375 in serum samples. The normalized data were analyzed using $t$-test or ANOVA, with asymptotic $P$ value computations at $P<0.05$ . Groups of patients with different miR-375 levels in their sera were evaluated by the two-tailed nonparametric MannWhitney $U$ test. Furthermore, Spearman correlation was used to analyze the correlation between the expression level of miR-375 and patient characteristics. ROC curve analysis was employed to determine the diagnostic utility of miR375. For cytological experiments, differences among experimental groups were statistically evaluated using $t$-tests. Sta- tistical analyses were performed using SPSS version 19.0 (IBM, USA). All graphs were constructed using GraphPad Prism 5 (GraphPad Software, USA).

\section{Results}

3.1. Description and Clinical Features of Patients. A total of 177 participants were enrolled in the study at the Department of Hepatobiliary Surgery, Xijing Hospital and the Center of Infectious Diseases, Tangdu Hospital from August 2015 to August 2017. A total of $63 \mathrm{HBV}-\mathrm{HCC}$ patients, 74 patients with $\mathrm{CHB}$, and 40 healthy controls were enrolled in this study. Among the 74 patients with $\mathrm{CHB}, 54$ had cirrhosis, including 20 patients with compensated cirrhosis and 34 patients with decompensated cirrhosis. The average age of all the subjects was $47.24 \pm 10.72$ years. The average age of the healthy controls and $\mathrm{HBV}$-infected subjects was 45.63 \pm 9.43 years and $48.99 \pm 10.93$ years, respectively, $80 \%$ $(32 / 40)$ of the males in healthy controls and $81 \%(111 / 137)$ of males with those infected with HBV. There was no significant difference in the distribution of age and sex between the above two groups $(P>0.05)$. All patients were positive for HBsAg, and 62 patients were positive for HBV DNA. In addition, $1.5 \%$ of the patients were positive for anti-HBs antibodies, 39.4\% were positive for $\mathrm{HBeAg}$ antibodies, $40.9 \%$ were positive for anti-HBe antibodies, and $92.7 \%$ were positive for anti-HBc antibodies. There were 74 patients with elevated ALT levels. The tumor markers alpha-fetoprotein (AFP), carcinoembryonic antigen (CEA), and carbohydrate antigen 199 (CA19-9) were elevated in 72, 32, and 51 patients, respectively. None of the patients had any other type of liver disease, such as chronic hepatitis $\mathrm{C}$ infection, alcoholic liver disease, autoimmune liver disease, or metabolic liver disease.

3.2. Expression of $m i R-375$ in Serum. To investigate whether serum miR-375 levels are abnormally altered in healthy individuals, in patients with $\mathrm{CHB}$ with or without cirrhosis, and in patients with HCC, serum miR-375 levels were measured in 137 patients and 40 healthy controls. The expression of serum miR-375 by qRT-PCR analysis decreased in order from healthy controls to patients with $\mathrm{CHB}$ without cirrhosis, followed by patients with cirrhosis, and finally, patients with HBV-HCC. Compared with those in healthy controls, serum miR-375 levels were significantly decreased in cirrhosis and $\mathrm{HBV}-\mathrm{HCC}$ patients $(P<0.05)$. Among the $\mathrm{HBV}$ infected patients, serum miR-375 levels were significantly higher in the CHB group than those in the HBV-HCC group $(P<0.05)$ (Figure 1).

3.3. Relationship between Serum miR-375 Levels and $H B V$ Serological Markers in HBV-Infected Patients. To assess whether serum miR-375 levels were associated with viral replication-related and HBV serological markers, the correlation between serum miR-375 levels and concentrations of HBV DNA (45.26\%), HBeAg (39.42\%), anti-HBe (40.88\%), anti-HBc (92.70\%), and anti-HBs (1.46\%) antibodies were analyzed in $\mathrm{HBV}$-infected patients. The expression of miR375 was significantly lower in HBeAg-positive patients than that in $\mathrm{HBeAg}$-negative patients $(P<0.05)$. The expression 


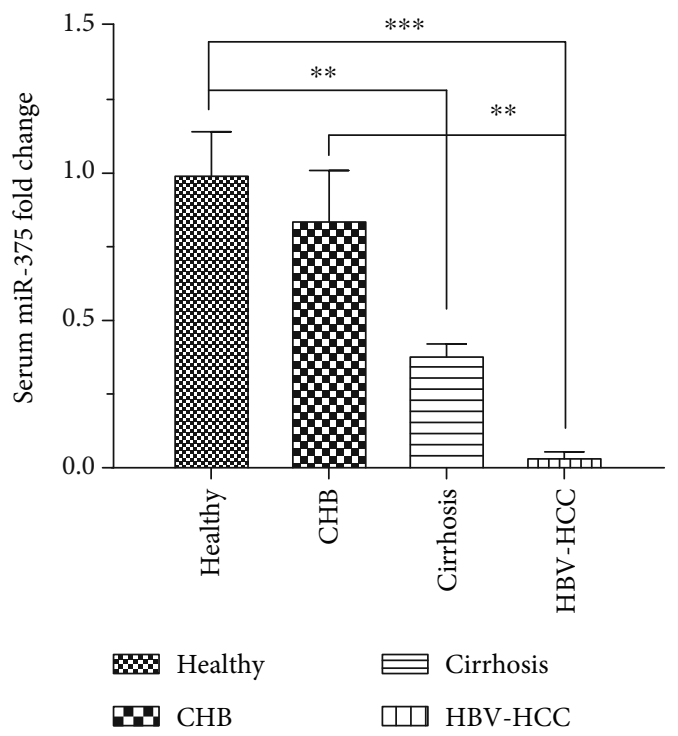

Figure 1: The differential serum levels of miR-375 in the HCC, cirrhosis, $\mathrm{CHB}$, and healthy control groups. The expression of serum miR-375 decreased in order from healthy controls to patients with chronic hepatitis $\mathrm{B}$ infection (CHB) without cirrhosis, followed by patients with $\mathrm{CHB}$ with cirrhosis (cirrhosis), and finally, patients with HBV-HCC. Serum miR-375 levels were significantly higher in healthy controls than in cirrhosis patients $\left({ }^{* *} P<0.01\right)$. Serum miR-375 levels were significantly higher in healthy controls than in HBV-HCC patients $\left({ }^{* * *} P<0.001\right)$. Serum miR-375 levels were significantly higher in patients with $\mathrm{CHB}$ than in patients with $\mathrm{HBV}-\mathrm{HCC}\left({ }^{* *} P<0.01\right)$.

of miR-375 was significantly lower in anti-HBe-negative patients than that in anti-HBc-positive patients $(P<0.05)$. Additionally, the expression of miR-375 was significantly lower in HBV DNA-positive patients than that in $\mathrm{HBV}$ DNA-negative patients $(P<0.05)$ (Table 1$)$.

3.4. Relationship between Serum miR-375 Levels and the Clinical Features of HBV-Infected Patients. We next analyzed the correlation between the expression of serum miR-375 and clinical features of patients, such as ALT, AFP, carbohydrate antigen 19-9 (CA19-9), and carcinoembryonic antigen (CEA) levels. The expression of miR-375 was significantly lower in patients with elevated AFP and CEA levels than that in patients with normal AFP and CEA levels $(P<0.05$, Table 2).

3.5. Diagnostic Value of Serum miR-375 for HBV-HCC. ROC curve analysis was performed to verify the accuracy of serum miR-375 in diagnosing HBV-HCC. Indeed, serum miR-375 levels could serve as valuable biomarkers for differentiating HCC patients from the whole cohort with an AUC of 0.838 (95\% confidence interval (CI): 0.780-0.897, Figure 2) and sensitivity and specificity of $73.9 \%$ and $93.0 \%$, respectively. We compared the diagnostic accuracy of miR-375 and AFP using the same serum samples (from $137 \mathrm{HBV}$-infected patients). miR-375 could differentiate HCC patients from other HBV-infected patients with an AUC of 0.768 (95\% CI: $0.644-0.891$, Figure 2), sensitivity of $93.8 \%$, and specificity
TABLE 1: Relationship between serum miR-375 levels and HBV serological markers in the HBV-infected patients $(n(\%))$.

\begin{tabular}{lcccc}
\hline $\begin{array}{l}\text { HBV serological } \\
\text { markers }\end{array}$ & $\begin{array}{c}\text { Number of } \\
\text { patients }(\%)\end{array}$ & miR-375 levels & $t$ value & $P$ \\
\hline $\begin{array}{c}\text { Anti-HBs } \\
\text { Positive }\end{array}$ & $2(1.46 \%)$ & $0.019 \pm 0.016$ & 0.662 & 0.509 \\
Negative & $135(98.54 \%)$ & $0.125 \pm 0.021$ & & \\
HBeAg & & & 2.037 & $0.044^{*}$ \\
Positive & $54(39.42 \%)$ & $0.081 \pm 0.020$ & & \\
Negative & $83(60.58 \%)$ & $0.186 \pm 0.047$ & & \\
Anti-HBe & & & 2.174 & $0.032^{*}$ \\
Positive & $56(40.88 \%)$ & $0.078 \pm 0.020$ & & \\
Negative & $81(59.12 \%)$ & $0.164 \pm 0.034$ & & \\
Anti-HBc & & & 0.961 & 0.339 \\
Positive & $127(92.70 \%)$ & $0.129 \pm 0.022$ & & \\
Negative & $10(7.30 \%)$ & $0.058 \pm 0.038$ & & \\
HBV DNA & & & 2.022 & $0.046^{*}$ \\
Positive & $62(45.26 \%)$ & $0.083 \pm 0.023$ & & \\
Negative & $75(54.74 \%)$ & $0.196 \pm 0.050$ & & \\
\hline
\end{tabular}

Serum HBV DNA $\geq 10^{2}$ copies/mL was defined as HBV DNA-positive. Serum HBV DNA $<10^{2}$ copies/mL was defined HBV DNA-negative. ${ }^{*} P<0.05$.

TABLE 2: Relationship between serum miR-375 levels and clinical features $(n(\%))$.

\begin{tabular}{lcccc}
\hline Clinical features & $\begin{array}{r}\text { Number of } \\
\text { patients (\%) }\end{array}$ & miR-375 levels & $t$ value & $P$ \\
\hline ALT & & & 0.363 & 0.717 \\
Elevated & $74(54.0 \%)$ & $0.103 \pm 0.027$ & & \\
Normal & $63(46.0 \%)$ & $0.116 \pm 0.026$ & & \\
AFP & & & 3.406 & $0.001^{* * *}$ \\
Elevated & $72(52.6 \%)$ & $0.048 \pm 0.015$ & & \\
Normal & $65(47.4 \%)$ & $0.252 \pm 0.058$ & & \\
CA19-9 & & & 1.100 & 0.273 \\
Elevated & $51(37.2 \%)$ & $0.102 \pm 0.029$ & & \\
Normal & $86(62.8 \%)$ & $0.170 \pm 0.044$ & & \\
CEA & & & 2.221 & $0.028^{*}$ \\
Elevated & $32(23.4 \%)$ & $0.063 \pm 0.030$ & & \\
Normal & $105(76.6 \%)$ & $0.170 \pm 0.038$ & & \\
\hline
\end{tabular}

Logistic regression analyses in subgroups of patients: categorized by serum levels of ALT $(<40 \mathrm{U} / \mathrm{L}$ and $\geq 40 \mathrm{U} / \mathrm{L})$, AFP $(<20 \mathrm{ng} / \mathrm{L}$ and $\geq 20 \mathrm{ng} / \mathrm{L})$, CA19-9 $(<37 \mathrm{U} / \mathrm{mL}$ and $\geq 37 \mathrm{U} / \mathrm{mL})$, and CEA $(<5 \mu \mathrm{g} / \mathrm{mL}$ and $\geq 5 \mu \mathrm{g} / \mathrm{mL})$ at baseline. ${ }^{*} P<0.05,{ }^{* * *} P<0.001$.

of $63.9 \%$. When we used AFP to separate HBV-HCC patients from $\mathrm{HBV}$-infected patients, the AUC was 0.584 (95\% CI: $0.456-0.713$ ), the sensitivity was $75.0 \%$, and the specificity was $65.5 \%$.

3.6. miR-375 Was Downregulated in HBV-HCC Cell Lines. HepG2.2.15, HepAD38, and parental HepG2 cells were 


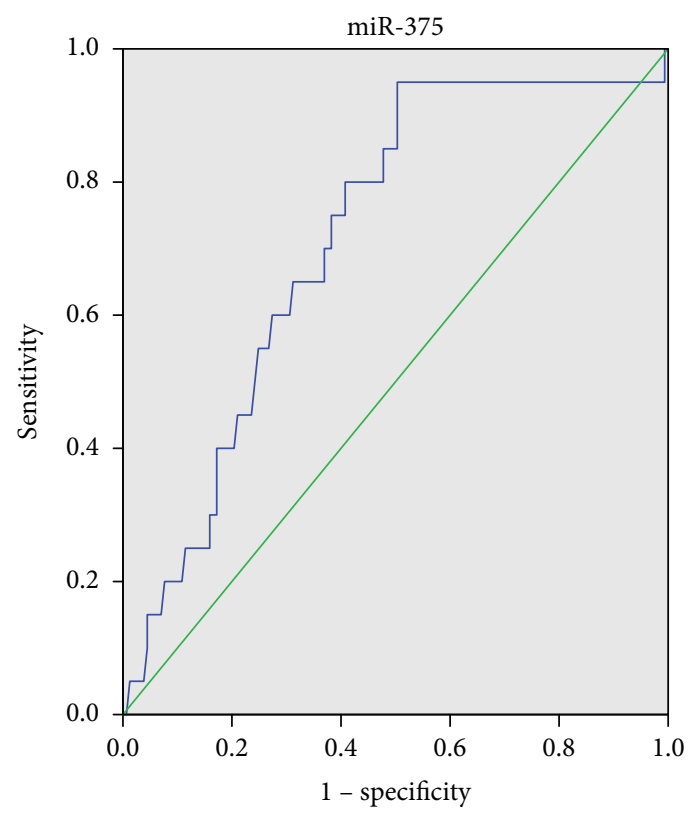

(a)

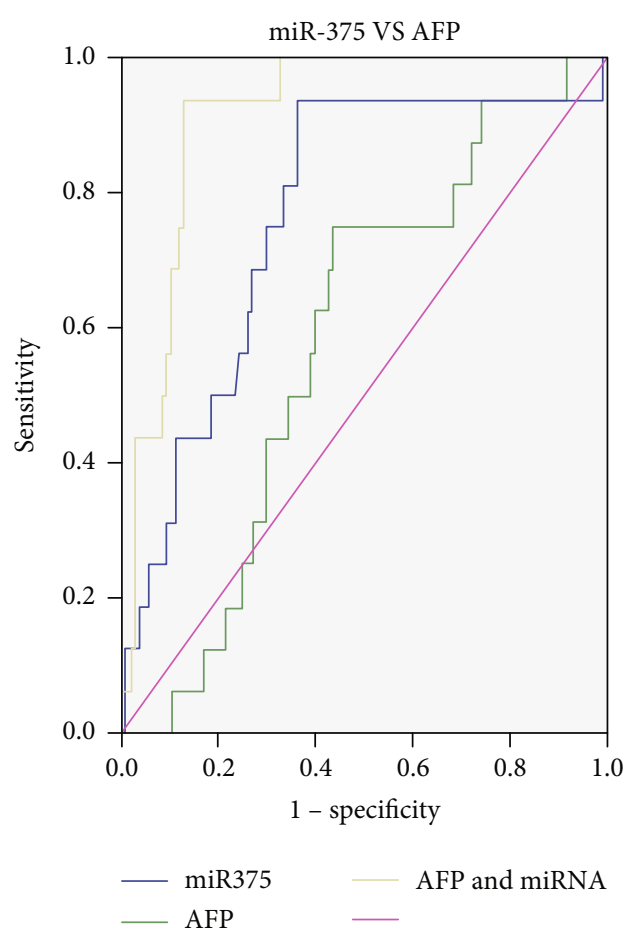

(b)

FIGURE 2: ROC curve analysis of the diagnostic value of serum miR-375 for HCC. (a) AUC estimation for the value of serum miR-375 in identifying HCC patients within the whole cohort $(\mathrm{AUC}=0.838$, sensitivity $=73.9 \%$, and specificity $=93.0 \%)$. (b) AUC estimation of the value of serum miR-375 in distinguishing HCC patients from HBV patients (AUC $=0.768$, sensitivity $=93.8 \%$, and specificity $=63.9 \%$ ). AUC estimation of the value of AFP in distinguishing HCC patients from HBV patients (AUC $=0.584$, sensitivity $=75.0 \%$, and specificity $=65.5 \%)$.

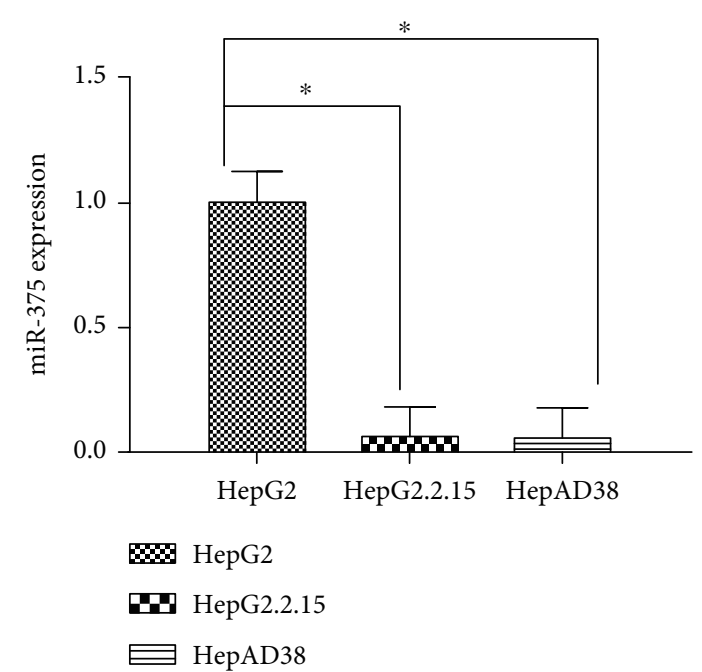

Figure 3: The expression of miR-375 in HepG2, HepG2.2.15, and HepAD38 cell lines. The expression of miR-375 was clearly lower in HepG2.2.15 cells and HepAD38 cells than that in HepG2 cells, respectively. ${ }^{*} P<0.05$.

selected for in vitro experiments. All cell lines were adjusted to $2 \times 10^{5} / \mathrm{mL}$ and collected to extract miRNA for use. HepG2.2.15 and HepAD38 cells were positive for HBsAg. However, HepG2 cells were negative for HBsAg, and $\mathrm{HBV}$ DNA copy numbers in these cells were below the level of detection. Based on qRT-PCR analysis, miR-375 expression was clearly lower in HepG2.2.15 cells and HepAD38 than that in HepG2 cells $(P<0.05$, Figure 3$)$, which is consistent with the results of serological analyses.

3.7. miR-375 Inhibited the Proliferation of HBV-HCC Cells. We tested the expression level of miR-375 by qRT-PCR in HepG2 cells, HepG2.2.15 cells, and HepAD38 cells at $48 \mathrm{~h}$ after transfection to determine the transfection efficiency. The expression of miR-375 in the transfected cells was significantly higher than that in the nontransfected control cells by nearly 15-20-folds, indicating that the expression of miR-375 in the cells was enhanced after transfection.

We performed the CCK-8 assay in HepG2 cells, HepG2.2.15 cells, and HepAD38 cells at $24 \mathrm{~h}, 48 \mathrm{~h}$, and $72 \mathrm{~h}$ after transfecting the cells with miR-375 mimics. The proliferation rates of miR-375-transfected cells were lower than those of nontransfected cells (transfected vs. nontransfected HepG2.2.15 cells, transfected vs. nontransfected HepAD38 cells, and transfected vs. nontransfected HepG2 cells). The proliferation rates of HepG2.2.15 cells and HepAD38 cells were lower than those of HepG2 cells (transfected HepG2.2.15 cells and HepAD38 vs. transfected HepG2cells, nontransfected HepG2.2.15 and HepAD38 cells vs. nontransfected HepG2 cells). The proliferation rates of transfected HepG2.2.15 cells and HepAD38 were the lowest among all groups. Therefore, the overexpression of miR375 significantly inhibited cellular proliferation (Figure 4). 


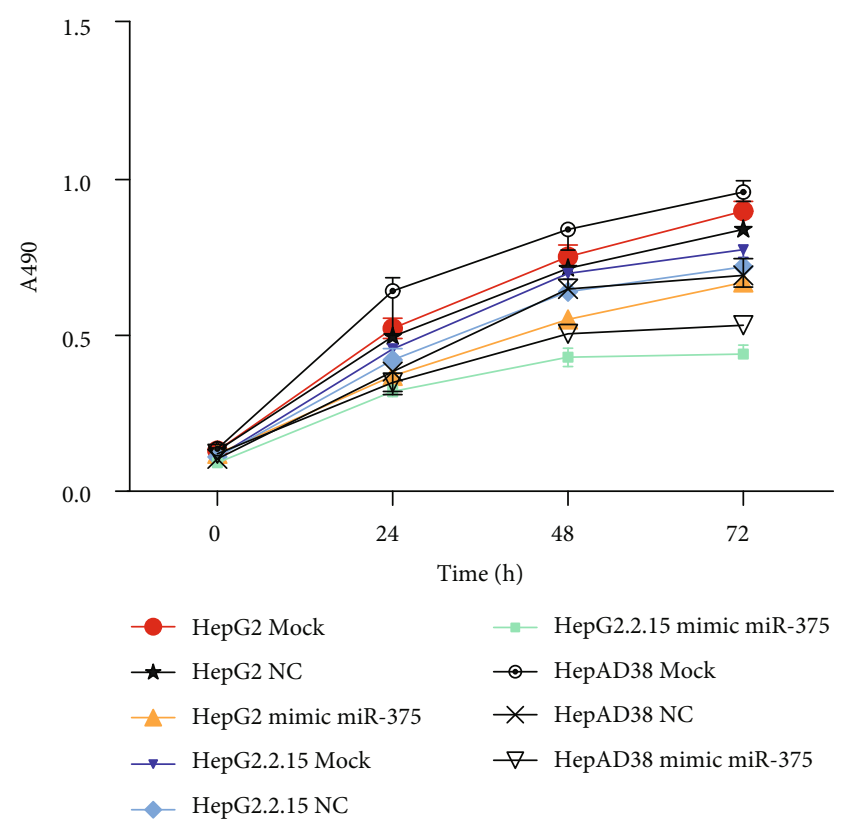

FIGURE 4: The effect of miR-375 on the proliferation of HCC cells. We performed the CCK- 8 assay in HepG2 cells, HepG2.2.15 cells, and HepAD38 cells at $24 \mathrm{~h}, 48 \mathrm{~h}$, and $72 \mathrm{~h}$ after transfecting the cells with miR-375 mimics. HepG2.2.15 and HepAD38 cells were positive for HBsAg. However, HepG2 cells were negative for HBsAg. The proliferation rates of miR-375-transfected cells were lower than those of nontransfected cells (transfected vs. nontransfected HepG2.2.15 cells, transfected vs. nontransfected HepAD38 cells, and transfected vs. nontransfected HepG2 cells). The proliferation rates of HepG2.2.15 cells and HepAD38 cells were lower than those of HepG2 cells (transfected HepG2.2.15 cells vs. transfected HepG2 cells, transfected HepAD38 cells vs. transfected HepG2 cells, and nontransfected HepG2.2.15 cells vs. nontransfected HepG2 cells).

\section{Discussion}

The purpose of the present study was to explore novel serum biomarkers for the prediction of HBV-HCC progression. Because of the lack of reliable serum biomarkers with high sensitivity and specificity, we conducted the present study to identify whether miR-375 has a potential diagnostic value in predicting HBV-related hepatitis or cirrhosis to HCC.

First, we analyzed serum miR-375 levels in healthy controls and in patients with CHB, cirrhosis, or HBV-HCC. Compared with those in healthy controls, serum miR-375 levels were significantly decreased in cirrhosis and HBVHCC patients $(P<0.05)$. These results were consistent with our previous study, which compared miR-375 expression in tumor and adjacent normal tissues from patients with HBV-HCC [26]. In that study, miR-375 levels were significantly lower in tumor tissues than in adjacent tissues from HBV-HCC patients. In other words, miR-375 expression is downregulated in both tissues and sera of HBV-HCC patients. Previous studies have also demonstrated that miR375 is significantly downregulated in multiple types of cancer and it plays the role of a tumor suppressor $[3,18]$. Moreover, our data showed that the expression of serum miR-375 decreased in order from healthy controls to patients with $\mathrm{CHB}$ without cirrhosis, followed by patients with cirrhosis, and finally, patients with HBV-HCC. Serum miR-375 expression was the lowest in the HBV-HCC group. Therefore, the expression of serum miR-375 decreases with disease progression. With the identification of the potential target genes of these HCC-associated miRNAs, it may shed light into our understanding of tumorigenesis and development of HCC. For example, serum miR-375 contributed to separation of both HBV-infection cases and HBV-positive HCC cases from the controls.

Next, we analyzed the relationship between serum miR375 levels and HBV serological markers in HBV-infected patients. Serum miR-375 expression was significantly lower in $\mathrm{HBeAg}$-positive and HBV DNA-positive patients than that in HBeAg-negative and HBV DNA-negative patients. HBV DNA is a quantitative virologic marker reflecting the degree of replication of HBV. A number of studies have found that the amount of HBV DNA is related to the extent of liver injury and severity of liver fibrosis and that it can be used as an independent factor to predict response to antiviral treatment [26-28]. $\mathrm{HBeAg}$ is a serologic marker associated with a high degree of viral replication and infectivity. In general, HBeAg positivity is correlated with a high HBV DNA load [29]. In this study, the expression of serum miR-375 was relatively lower in HBV DNA-positive and HBeAgpositive patients. The results of our previous study showed that the abnormal expression of miR-375 in HBV-HCC patient tissues was closely related to the replication status of $\mathrm{HBV}$. In addition, the expression level of miR-375 in tissues was negatively correlated with the HBV DNA load. The higher was the titer of HBV DNA, the lower was the expression level of miR-375 in tissues [26]. These results were consistent with the results of the current study.

Next, we analyzed the relationship between serum miR375 levels and commonly used serum tumor markers. AFP is one of the most widely used tumor markers for the diagnosis of HCC, and it has a sensitivity of $60 \%$ at a cutoff value of $20 \mathrm{ng} / \mathrm{mL}[4,27]$. A systematic review evaluating AFP (at a threshold level of $20 \mathrm{ng} / \mathrm{mL}$ ) in cirrhotic patients showed sensitivities and specificities of $41 \%$ to $65 \%$ and $80 \%$ to $94 \%$, respectively, for HCC at any stage [30]. However, at this threshold, early stage HCC was detected in only one-third of patients with the disease [31]. The problem with AFP as a reliable HCC biomarker is that HCC is positive for the protein in only $60 \%-80 \%$ of cases, and false positives make it difficult to distinguish early stage HCC from other disorders, such as acute hepatitis and cirrhosis, as well as embryonic tumors and certain gastrointestinal tumors. Thus, in order to significantly improve the diagnostic accuracy for HCC, additional biomarkers are needed to complement AFP, especially due to the fact that many patients with benign liver diseases, such as chronic hepatitis, liver cirrhosis, and gastrointestinal cancer, also have elevated serum AFP. To compare the diagnostic accuracy of miR-375 and AFP, we analyzed the AUC of the two markers using the same serum samples. The AUC (0.768 vs. 0.584 ) and sensitivity ( $93.8 \%$ vs. $75.0 \%)$ of serum miR-375 were higher than those for AFP. Therefore, serum miR-375 is better than AFP for diagnosing 
HBV-HCC. Nevertheless, these results need to be validated in larger cohorts in the future.

In recent years, some studies have shown that abnormal expression of miR-375 can affect the proliferation, migration, and invasion of HCC cell lines, including Hep3B and Huh7 $[24,32]$. However, these studies determined the expression of miR-375 and its downstream regulatory genes in pure hepatoma cell lines but not HBV-HCC cell lines. Therefore, the effect of the abnormal expression of miR-375 on the biological function of HBV-HCC cell lines remained unknown. The cell lines HepG2.2.15 and HepAD38 that were used in this study have been derived by the stable expression of HBV in HepG2 cells. We routinely cultured and confirmed the identity of HepG2, HepG2.2.15, and HepAD38 cells. HepG2.2.15 and HepAD38 cells were positive for HBsAg, and HBV DNA titers in these cells indicated moderate viral replication, whereas HepG2 cells were negative for $\mathrm{HBsAg}$ and $\mathrm{HBV}$ DNA. Therefore, HepG2.2.15 and HepAD38 displayed characteristics of HBV infection, and there was no cross contamination between the three cell lines.

In vitro, we determined the expression level of miR-375 in HepG2.2.15, HepAD38 cells, and parental HepG2 cells using qRT-PCR. Compared with that in HepG2 cells, which are not infected with HBV, the expression level of miR-375 was clearly reduced in HepG2.2.15 cells and HepAD38, which are infected with HBV. In other words, the expression level of miR-375 in liver cancer cells is affected by HBV infection. Some studies have also shown that other miRNAs are differentially expressed between cell lines with stable HBV infection and their parental counterparts [26]. miRNAs play a critical role in virus-host interactions. Cellular miRNAs in the host modulate the expression of various viral genes, thus playing a pivotal role in the host-pathogen interaction network. In addition, viruses also encode miRNAs for protection against cellular antiviral responses or may even exploit host miRNA pathways to their own advantage. The persistence of the virus in host cells in turn affects the expression of miRNAs in the host.

We upregulated miR-375 expression in HepG2, HepG2.2.15, and HepAD38 cells and analyzed the effects of miR-375 overexpression on proliferation and invasion in vitro using the CCK-8 and transwell assays. The overexpression of miR-375 significantly inhibited cellular proliferation. In HepG2, HepG2.2.15, and HepAD38 cells, the proliferation rate of miR-375-transfected cells was lower than that of nontransfected cells. Maximum inhibition was observed at $48 \mathrm{~h}$ posttransfection. Additionally, the proliferation rates of HepG2.2.15 cells and HepAD38 with or without miR-375 overexpression were significantly lower than the respective proliferation rates of HepG2 cells with or without miR-375 overexpression. Between 48 and $72 \mathrm{~h}$ posttransfection with miR-375, the size of the HepG2.2.15 cell population remained almost constant. Therefore, miR-375 could inhibit the proliferation of liver cancer cells, and the inhibition was more pronounced in the presence of HBV infection.

In other words, miR-375 plays a similar tumor suppressor gene. Based on past experiments, JAK-2 acts as a downstream target of miR-375 and is regulated by miR-375, which affects the normal expression of genes and proteins in the signal transduction pathway and affects the proliferation and invasion of tumor cells. Similarly, YAP1 is also an important target molecule of miR-375, which is an important molecule in the hippo signaling pathway. It can promote proliferation and growth and plays an important role in DNA repair. When the hippo pathway abnormal YAP1 is activated, YAP1 is a famous oncoprotein, and the incidence of cancer is closely related to the development of the tumor can promote. miR-375 can reverse the inhibition of YAP1 expression, so that YAP1 to promote the tumor was cut off to this channel to achieve the purpose of inhibiting tumorigenesis [33]. Because miR-375 likely serve a role in HCC development following chronic HBV infection, future study may focus on their target genes and the mechanisms by which they execute their functions during HBV infection and HBV-positive HCC development.

The present study has two limitations. First, during the transition from healthy liver, $\mathrm{HBC}$, and liver cirrhosis to HBV-HCC, there is an asymptomatic phase of HBV infection. However, our cohort did not include such HBV carriers. In the future, we will further expand the sample size and evaluate patients at various stages of $\mathrm{HBV}$ infection, including chronic asymptomatic HBV carriers. Thus, we will perform in-depth analysis to further confirm the diagnostic value of serum miR-375 for HBV-HCC. Second, this study only examined miR-375, and other potentially relevant markers, including some classic HCC risk factors, could not be evaluated. In the future, we will assess these markers together with miR-375 and construct a predictive model for HBV-HCC.

\section{Conclusion}

Serum miR-375 expression decreased in order from healthy controls to patients with $\mathrm{CHB}$ without cirrhosis, followed by patients with $\mathrm{CHB}$ with cirrhosis, and finally, patients with HBV-HCC. Serum miR-375 expression was correlated with $\mathrm{HBeAg}, \mathrm{HBV}$ DNA, and AFP and CEA levels. ROC curve analyses revealed that serum miR-375 may be a promising biomarker for HBV-HCC detection, with a relatively high AUC, sensitivity, and specificity. The AUC and sensitivity of serum miR- 375 were higher than those for AFP. In addition, compared with that in HepG2 cells, miR-375 expression was markedly reduced in HepG2.2.15 and HepAD38 cells. The overexpression of miR-375 clearly inhibited proliferation and migration in vitro, especially in HepG2.2.15 cells and HepAD38, which are stably infected with HBV. Therefore, serum miR-375 might serve as a potential biomarker to predict the progression of HBVrelated hepatitis or cirrhosis to HCC.

\section{Data Availability}

All data used to support the findings of this study are included within the article. The raw data in this study are restricted by the Independent Ethics Committee, The Air Force Medical University, China, in order to protect patient privacy. Data are available from the corresponding author: Zhongjun Shao (e-mail: zhongjunshao@126.com) 
for researchers who meet the criteria for access to confidential data.

\section{Conflicts of Interest}

The authors declare no conflicts of interest for this article.

\section{Authors' Contributions}

The first three authors contributed equally to this paper. WLZ, TF, and ZJG contributed in study concept and design, acquisition of data, analysis and interpretation of data, drafting of the manuscript, and obtaining funding; YZ, LZ, HXS, YL, JG, and ZHJ contributed in the acquisition of data, technical support, and analysis of data; YPY and ZJS contributed in study concept and design, critical revision of the manuscript for important intellectual content, and obtaining funding.

\section{Acknowledgments}

This study was funded by the National Natural Science Foundation of China (81773488, 81472988), the National Key R\&D Program of China (2016YFC1303204), and the National Key Project for Prevention of Infectious Disease (2017ZX10105011).

\section{References}

[1] World Health Organization, Guidelines for the prevention, care and treatment of persons with chronic hepatitis B infection, World Health Organization, Geneva, 2015.

[2] W. Chen, R. Zheng, P. D. Baade et al., "Cancer statistics in China, 2015," CA: a Cancer Journal for Clinicians, vol. 66, no. 2, pp. 115-132, 2016.

[3] A. M. Liu, R. T. Poon, and J. M. Luk, "MicroRNA-375 targets hippo-signaling effector YAP in liver cancer and inhibits tumor properties," Biochemical and Biophysical Research Communications, vol. 394, no. 3, pp. 623-627, 2010.

[4] J. Yin, P. Hou, Z. Wu, T. Wang, and Y. Nie, "Circulating miR375 and miR-199a-3p as potential biomarkers for the diagnosis of hepatocellular carcinoma," Tumour Biology, vol. 36, no. 6, pp. 4501-4507, 2015.

[5] S. W. Nam, K. C. Park, K. J. Yang, B. Lee, and S. W. Kim, "Genetic screen identifies suppressor of morphogenesis in genitalia-1 (SMG-1) as a modulator of sorafenib resistance in hepatocellular carcinoma cell lines," International Journal of Oncology, vol. 45, no. 4, pp. 1450-1456, 2014.

[6] L. L. Han, H. C. Nan, T. Tian et al., "Expression and significance of the novel tumor-suppressor gene SMG-1 in hepatocellular carcinoma," Oncology Reports, vol. 31, no. 6, pp. 2569-2578, 2014.

[7] D. Campion, A. Tucci, P. Ponzo, and G. P. Caviglia, "Noninvasive biomarkers for the detection of hepatocellular carcinoma," Minerva Biotecnologica, vol. 31, no. 1, pp. 11-22, 2019.

[8] Y. Zhang, J. X. Zhang, L. L. Huang et al., "Low expression of BARX2 in human primary hepatocellular carcinoma correlates with metastasis and predicts poor prognosis," Hepatology Research, vol. 45, no. 2, pp. 228-237, 2015.

[9] Q. F. Qin, J. Weng, G. X. Xu, C. M. Chen, and C. K. Jia, “Combination of serum tumor markers dickkopf-1, DCP and AFP for the diagnosis of primary hepatocellular carcinoma," Asian Pacific Journal of Tropical Medicine, vol. 10, no. 4, pp. 409413, 2017.

[10] B. Sun, Z. Huang, B. Wang et al., "Significance of glypican-3 (GPC3) expression in hepatocellular cancer diagnosis," Medical Science Monitor, vol. 23, pp. 850-855, 2017.

[11] J. P. Yu, X. G. Xu, R. J. Ma et al., "Development of a clinical chemiluminescent immunoassay for serum GPC3 and simultaneous measurements alone with AFP and CK19 in diagnosis of hepatocellular carcinoma," Journal of Clinical Laboratory Analysis, vol. 29, no. 2, pp. 85-93, 2015.

[12] J. Zhao, L. Y. Guo, J. M. Yang, and J. W. Jia, "Sublingual vein parameters, AFP, AFP-L3, and GP73 in patients with hepatocellular carcinoma," Genetics and Molecular Research, vol. 14, no. 2, pp. 7062-7067, 2015.

[13] J. Lou, L. Zhang, S. Lv, C. Zhang, and S. Jiang, "Biomarkers for hepatocellular carcinoma," Biomark Cancer, vol. 9, pp. 1-9, 2017.

[14] G. P. Caviglia, M. L. Abate, S. Gaia et al., "Risk of hepatocellular carcinoma in HBV cirrhotic patients assessed by the combination of miR-122, AFP and PIVKA-II," Panminerva Medica, vol. 59, no. 4, pp. 283-289, 2017.

[15] J. Balogh, D. Victor, E. H. Asham et al., "Hepatocellular carcinoma: a review," Journal of Hepatocellular Carcinoma, vol. Volume 3, pp. 41-53, 2016.

[16] B. Luo, N. Kang, Y. Chen, L. Liu, and Y. Zhang, "Oncogene miR-106a promotes proliferation and metastasis of prostate cancer cells by directly targeting PTEN in vivo and in vitro," Minerva Medica, vol. 109, no. 1, pp. 24-30, 2018.

[17] J. Lin, J. Xia, C. Z. Tu, K. Y. Zhang, Y. Zeng, and Q. Yang, "H9N2 avian influenza virus protein PB1 enhances the immune responses of bone marrow-derived dendritic cells by down-regulating miR375," Frontiers in Microbiology, vol. 8, p. 287, 2017.

[18] J. W. Yan, J. S. Lin, and X. X. He, "The emerging role of miR375 in cancer," International Journal of Cancer, vol. 135, no. 5 , pp. 1011-1018, 2014.

[19] B. Zhan, D. Lu, P. Luo, and B. Wang, "Prognostic value of expression of microRNAs in non-small cell lung cancer: a systematic review and meta-analysis," Clinical Laboratory, vol. 62, no. 11, pp. 2203-2211, 2016.

[20] M. Winther, J. Alsner, T. Tramm, L. Baeksgaard, E. Holtved, and M. Nordsmark, "Evaluation of miR-21 and miR-375 as prognostic biomarkers in esophageal cancer," Acta Oncologica, vol. 54, no. 9, pp. 1582-1591, 2015.

[21] Y. Shao, Y. Geng, W. Gu, J. Huang, Z. Ning, and H. Pei, “Prognostic significance of microRNA-375 downregulation in solid tumors: a meta-analysis," Disease Markers, vol. 2014, Article ID 626185, 10 pages, 2014.

[22] N. Zhou, J. Wu, X. Wang, Z. Sun, Q. Han, and L. Zhao, "Lowlevel expression of microRNA-375 predicts poor prognosis in hepatocellular carcinoma," Tumour Biology, vol. 37, no. 2, pp. 2145-2152, 2016.

[23] L. M. Li, Z. B. Hu, Z. X. Zhou et al., "Serum microRNA profiles serve as novel biomarkers for HBV infection and diagnosis of HBV-positive hepatocarcinoma," Cancer Research, vol. 70, no. 23, pp. 9798-9807, 2010.

[24] Y. Chang, W. Yan, X. He et al., “miR-375 inhibits autophagy and reduces viability of hepatocellular carcinoma cells under hypoxic conditions," Gastroenterology, vol. 143, no. 1, pp. 177-87.e8, 2012. 
[25] Z. Liu, C. Ma, X. Tang et al., "The reciprocal interaction between LncRNA CCAT1 and miR-375-3p contribute to the downregulation of IRF5 gene expression by solasonine in HepG2 human hepatocellular carcinoma cells," Frontiers in Oncology, vol. 9, p. 1081, 2019.

[26] T. Fu, K. Liu, W. L. Zhang et al., "miR-375 expression in tumor and adjacent normal tissues of patient with hepatitis B virusassociated hepatocellular carcinoma," Progress in Modern Biomedicine, vol. 2, pp. 271-274, 2016.

[27] F. M. Sanai, A. Helmy, K. I. Bzeizi et al., "Discriminant value of serum HBV DNA levels as predictors of liver fibrosis in chronic hepatitis B," Journal of Viral Hepatitis, vol. 18, no. 7, pp. e217-e225, 2011.

[28] S. Tong and P. Revill, "Overview of hepatitis B viral replication and genetic variability," Journal of Hepatology, vol. 64, 1 Suppl, pp. S4-16, 2016.

[29] F. J. Rabbi, M. K. Rezwan, and T. Shirin, "HBeAg/anti-HBe, alanine aminotransferase and HBV DNA levels in HBsAg positive chronic carriers," Bangladesh Medical Research Council Bulletin, vol. 34, no. 2, pp. 39-43, 2008.

[30] S. Gupta, S. Bent, and J. Kohlwes, "Test characteristics of alpha-fetoprotein for detecting hepatocellular carcinoma in patients with hepatitis C. A systematic review and critical analysis," Annals of Internal Medicine, vol. 139, no. 1, pp. 46-50, 2003.

[31] F. Trevisani, P. E. D'Intino, A. M. Morselli-Labate et al., "Serum $\alpha$-fetoprotein for diagnosis of hepatocellular carcinoma in patients with chronic liver disease: influence of HBsAg and anti-HCV status," Journal of Hepatology, vol. 34, no. 4, pp. 570-575, 2001.

[32] H. B. El-Serag, J. A. Marrero, L. Rudolph, and K. R. Reddy, "Diagnosis and treatment of hepatocellular carcinoma," Gastroenterology, vol. 134, no. 6, pp. 1752-1763, 2008.

[33] L. Miao, K. Liu, M. Xie, Y. Xing, and T. Xi, "miR-375 inhibits Helicobacter pylori-induced gastric carcinogenesis by blocking JAK2-STAT3 signaling," Cancer Immunology, Immunotherapy, vol. 63, no. 7, pp. 699-711, 2014. 